\title{
A one-shot proof of Arrow's theorem and the Gibbard-Satterthwaite theorem
}

\author{
Ning Neil Yu
}

Received: 12 June 2013 / Accepted: 28 June 2013 / Published online: 9 July 2013

(C) SAET 2013

\begin{abstract}
This paper provides a simple and transparent proof of a new social choice impossibility theorem. The Gibbard-Satterthwaite theorem and Arrow's impossibility theorem are straightforward corollaries.
\end{abstract}

Keywords Arrow's impossibility theorem - Gibbard-Satterthwaite theorem • Social choice and welfare

JEL Classification $\quad$ D7 $\cdot$ D70 $\cdot$ D71

\section{Introduction}

One of the impossibility theorems introduced by Yu (2013) can help prove both the Gibbard-Satterthwaite theorem (Gibbard 1973; Satterthwaite 1975) and Arrow's impossibility theorem (Arrow 1963) succinctly. In this paper, we offer a direct proof of this theorem, which resembles Yu (2012) and employs the "pivotal voter" technique devised and perfected by Barberá (1980), Geanakoplos (2005), and Reny (2001). We comment on different approaches of establishing classical theorems in the last section.

\section{The setup}

The following terminology follows Yu (2013) closely.

A set of individuals $\mathcal{N}=\{1, \ldots, N\}$ with $N \geq 2$, each have some preferences over $M \geq 3$ alternatives $A=\left\{a_{1}, \ldots, a_{M}\right\}$. Throughout the paper, we label individuals

N. N. Yu (凶)

Economics Department, Stanford University, 579 Serra Mall, Stanford, CA 94305-6072, USA

e-mail:ningyu@stanford.edu 
with $n$ and number alternatives with $i, j, k$. Let $\mathcal{P}$ be the set of all possible relations on $M$ that are complete, asymmetric, and transitive, i.e., strict preference relations. ${ }^{1}$ A preference profile is an ordered list $\vec{\succ}=\left(\succ_{1}, \ldots, \succ_{N}\right)$ with $\succ_{n} \in \mathcal{P}$ for every $1 \leq n \leq N$. All possible preference profiles form the collection $\mathcal{P}^{\mathcal{N}}$.

Definition $1(S C F)$ A social choice function $F: \mathcal{P}^{\mathcal{N}} \rightarrow A$ assigns to each $\vec{\succ} \in \mathcal{P}^{\mathcal{N}}$ a choice $F(\vec{\succ}) \in A$.

Definition 2 (SPF) A social preference function $R: \mathcal{P}^{\mathcal{N}} \rightarrow \mathcal{P}$ assigns to each $\vec{\succ} \in$ $\mathcal{P}^{\mathcal{N}}$ a preference relation $R(\vec{\succ}) \in \mathcal{P}$.

Several definitions facilitate communication.

Definition 3 For profile $\vec{\succ}, a_{i}$ dominates $a_{j}$ if $a_{i} \succ_{n} a_{j}$ for every $n$.

Definition 4 Two profiles $\vec{\succ}$ and $\vec{\succ}^{\prime}$ agree on $\left\{a_{i}, a_{j}\right\}$ if $a_{i} \succ_{n} a_{j}$ is necessary and sufficient for $a_{i} \succ_{n}^{\prime} a_{j}$ for every $n$.

Definition $5 A^{\prime} \subset A$ is at the top of $\vec{\succ}$ if $a_{i} \succ_{n} a_{j}$ for every $n$, every $a_{i} \in A^{\prime}$, and every $a_{j} \in A \backslash A^{\prime}$. If in addition $A^{\prime}=\left\{a_{i}, a_{j}\right\}$, we call $\vec{\succ}$ a $\{i, j\}$-runoff.

Definition 6 A runoff generating function $T_{i j}: \mathcal{P}^{\mathcal{N}} \rightarrow \mathcal{P}^{\mathcal{N}}$ brings $A^{\prime}=\left\{a_{i}, a_{j}\right\}$ to the top, keeping internal rankings of $A^{\prime}$ and $A \backslash A^{\prime}$ intact.

\section{The main theorem}

Definition $7(D)$ A SCF $F$ is dictatorial if there exists a social choice dictator $n$ such that $F(\vec{\succ}) \succ_{n} a_{i}$ for every $a_{i} \neq F(\vec{\succ})$ and every $\vec{\succ}$.

Definition $8(O)$ A SCF $F$ is onto if $F\left(\mathcal{P}^{\mathcal{N}}\right)=A$.

Definition $9(W P)$ A SCF $F$ is weakly Paretian if $a_{i}$ dominating $a_{j}$ implies $F(\vec{\succ}) \neq$ $a_{j}$ for every $i, j$ and every $\vec{\succ}$.

Definition $10(C M)$ A SCF $F$ is Condorcet monotonic if whenever $\vec{\succ}$ and $\{i, j\}$-runoff $\vec{\succ}^{\prime}$ agree on $\left\{a_{i}, a_{j}\right\}, F(\vec{\succ})=a_{i}$ implies $F\left(\vec{\succ}^{\prime}\right)=a_{i}$.

This condition requires every social choice to be a generalized Condorcet win$n e r$ in that it has to win any runoff it enters that keeps intact its rankings against the opponent. Losing a $\{i, j\}$-runoff prevents $a_{i}$ from winning any profile that agrees with it on $\left\{a_{i}, a_{j}\right\}$, no matter what rule-for example, simple majority, super majority, or weighted majority-determines the runoff outcome. In particular, if $F(\vec{\succ})=$ $a_{i}, F\left(T_{i j}(\vec{\succ})\right)=a_{i}$ by $(\mathrm{CM})$. We often apply the contrapositive statement.

Lemma 1 (O) and (CM) imply (WP).

Proof Let $a_{i}$ dominate $a_{j}$ in $\vec{\succ}$. (O) ensures that $F\left(\vec{\succ}^{\prime}\right)=a_{i}$ for some $\vec{\succ}^{\prime}$. By (CM), the choice remains $a_{i}$ for $T_{i k}\left(\vec{\succ}^{\prime}\right)$, where $k \notin\{i, j\}$, and further for $T_{i j}\left(T_{i k}\left(\vec{\succ}^{\prime}\right)\right)$. Since

\footnotetext{
1 The proofs that deal with weak preferences are published on my personal website: http://sites.google. com/site/neilningyu/.
} 
$a_{j}$ loses the $\{i, j\}$-runoff $T_{i j}\left(T_{i k}\left(\vec{\succ}^{\prime}\right)\right)$ that agrees with $\vec{\succ}$ on $\left\{a_{i}, a_{j}\right\}$, the choice for $\vec{\succ}$ cannot be $a_{j}$.

Theorem 1 If a SCF F satisfies (O) and (CM), then it is dictatorial.

Proof Pick any $\vec{\succ}^{\prime}$ with $\left\{a_{i}\right\}$ and $\left\{a_{i}, a_{j}\right\}$ at the top. Swap the positions of $\left\{a_{i}, a_{j}\right\}$ sequentially from 1 to $N$. By (WP), the choice is either $a_{i}$ or $a_{j}$, starting with $a_{i}$ and ending with $a_{j}$. The $(i, j)$-pivotal voter $n_{i j}$ is the first whose swap makes a difference. By (CM), any two $(i, j)$-runoffs that agree on $\left\{a_{i}, a_{j}\right\}$ select the same alternative, so $n_{i j}$ is independent of which $\vec{\succ}^{\prime}$ (with $\left\{a_{i}\right\}$ and $\left\{a_{i}, a_{j}\right\}$ at the top) we start with.

\begin{tabular}{l|lllllll} 
& 1 & $\ldots$ & $n_{i j}-1$ & $n_{i j}$ & $n_{i j}+1$ & $\ldots$ & $N$ \\
\hline$\vec{\succ}^{\prime \prime}$ & $a_{j}$ & $\ldots$ & $a_{j}$ & $a_{i}$ & $a_{i}$ & $\ldots$ & $a_{i}$ \\
& $\square$ & $\ldots$ & $\square$ & & & & \\
& $a_{i}$ & $\ldots$ & $a_{i}$ & $a_{j}$ & $a_{j}$ & $\ldots$ & $a_{j}$ \\
& $\square$ & $\ldots$ & $\square$ & $a_{k}$ & $a_{k}$ & $\ldots$ & $a_{k}$ \\
\hline$\vec{\succ}^{\prime \prime \prime}$ & $\square$ & $\ldots$ & $\square$ & & & & \\
& $a_{j}$ & $\ldots$ & $a_{j}$ & $a_{j}$ & $a_{i}$ & $\ldots$ & $a_{i}$ \\
& $\square$ & $\ldots$ & $\square$ & & $\square$ & $\ldots$ & $\square$ \\
& $a_{i}$ & $\ldots$ & $a_{i}$ & $a_{i}$ & $a_{j}$ & $\ldots$ & $a_{j}$ \\
& $\square$ & $\ldots$ & $\square$ & $a_{k}$ & $\square$ & $\ldots$ & $\square$
\end{tabular}

Consider depicted profiles $\vec{\succ}^{\prime \prime}$ and $\vec{\succ}^{\prime \prime \prime}$ with $\left\{a_{i}, a_{j}, a_{k}\right\}$ at the top, where columns correspond to voters and squares mark possible positions of $a_{k}$. The definition of $n_{i j}$ informs us that $F\left(T_{i j}\left(\vec{\succ}^{\prime \prime}\right)\right)=a_{i}$ and $F\left(T_{i j}\left(\vec{\succ}^{\prime \prime \prime}\right)\right)=a_{j}$, so $F\left(\vec{\succ}^{\prime \prime}\right) \neq a_{j}$ and $F\left(\vec{\succ}^{\prime \prime \prime}\right) \neq a_{i}$ by $(\mathrm{CM})$. For $\vec{\succ}^{\prime \prime}$, the choice is $a_{i}$, for (WP) rules out $a_{k}$ and others. Hence, $F\left(T_{i k}\left(\vec{\succ}^{\prime \prime}\right)\right)=a_{i}$, implying that in defining $n_{i k}$, no swap before $n_{i j}$ makes a difference, i.e., $n_{i k} \geq n_{i j}$. But $j$ and $k$ are arbitrary, so $n_{i j}=n_{i k}$, i.e., $n_{i-}$ refers to the same individual.

Moreover, $T_{i k}\left(\vec{\succ}^{\prime \prime}\right)$ and $\vec{\succ}^{\prime \prime \prime}$ agree on $\left\{a_{i}, a_{k}\right\}$, so $F\left(\vec{\succ}^{\prime \prime \prime}\right)$ is not $a_{k}$ due to its loss to $a_{i}$ in $\{i, k\}$-runoff $T_{i k}\left(\vec{\succ}^{\prime \prime}\right)$. We are left with $F\left(\vec{\succ}^{\prime \prime \prime}\right)=a_{j}$, so $F\left(T_{j k}\left(\vec{\succ}^{\prime \prime \prime}\right)\right)=a_{j}$, demanding by $(\mathrm{CM})$ that

$$
a_{j} \succ_{n_{i j}} a_{k} \text { implies } F(\vec{\succ}) \neq a_{k}
$$

In defining $n_{j k}$, $(*)$ says that no swap before $n_{i j}$ makes a difference, so $n_{j k} \geq n_{i j}$ or $n_{j-} \geq n_{i-}$. But $i$ and $j$ are arbitrary, confirming $n_{j-}=n_{i-}$. The single pivotal voter can eliminate any alternative except her favorite by $(*)$.

\section{Classical impossibility theorems}

Given Theorem 1, we can prove the Gibbard-Satterthwaite theorem through a simple lemma. Let $\left(\succ_{n}^{\prime}, \vec{\succ}_{-n}\right)$ represent the new profile constructed by replacing the preferences of individual $n$ in $\vec{\succ}$ with $\succ_{n}^{\prime} \in \mathcal{P}$. 
Definition $11(S P)$ A SCF $F$ is strategy-proof if $F\left(\succ_{n}^{\prime}, \vec{\succ}_{-n}\right) \neq F(\vec{\succ})$ implies $F(\vec{\succ}) \succ_{n} F\left(\succ_{n}^{\prime}, \vec{\succ}_{-n}\right)$ for every $n$, every $\vec{\succ}$, and every $\succ_{n}^{\prime}$.

This condition demands that an individual is worse off misreporting her preferences whenever a misreport can influence the choice, so truth-telling is optimal.

Lemma 2 (SP) imply (CM).

Proof Let $\vec{\succ}$ and $\{i, j\}$-runoff $\vec{\succ}^{\prime}$ agree on $\left\{a_{i}, a_{j}\right\}$. Suppose that $F(\vec{\succ})=a_{i}$ and $F\left(\succ_{1}^{\prime}, \vec{\succ}_{-1}\right)=a_{k}$ for $k \neq i$. By (SP), $a_{i} \succ_{1} a_{k}$. Define $\vec{\succ}^{\prime \prime}=\left(\succ_{1}^{\prime}, \vec{\succ}_{-1}\right)$. Given $\vec{\succ}=\left(\succ_{1}, \vec{\succ}_{-1}^{\prime \prime}\right)$, (SP) entails $a_{k} \succ_{1}^{\prime} a_{i}$. Since $\vec{\succ}^{\prime}$ is a $\{i, j\}$-runoff, $k=j$, contradicting the agreement of $\vec{\succ}$ and $\vec{\succ}^{\prime}$ on $\left\{a_{i}, a_{j}\right\}$. So $F\left(\succ_{1}^{\prime}, \vec{\succ}_{-1}\right)$ has to remain $a_{i}$. Likewise, the process of updating $\vec{\succ}$ to $\vec{\succ}^{\prime}$ one by one keeps the choice unaltered.

Theorem 2 (Gibbard-Satterthwaite) If a SCF F satisfies (O) and (SP), then it is dictatorial.

Proving Arrow's theorem is equally simple.

Definition $12(A D)$ A SPF $R$ is Arrow dictatorial if there exists a social preference dictator $n$ such that $a_{i} \succ_{n} a_{j}$ implies $a_{i} R(\vec{\succ}) a_{j}$ for every $i, j$ and every $\vec{\succ}$.

Definition $13(A U)$ A SPF $R$ is Arrow unanimous if $a_{i} \succ_{n} a_{j}$ for every $n$ implies $a_{i} R(\vec{\succ}) a_{j}$ for every $i, j$ and every $\vec{\succ}$.

Definition 14 (AIIA) A SPF $R$ is Arrow independent of irrelevant alternatives if whenever $\vec{\succ}$ and $\vec{\succ}^{\prime}$ agree on $\left\{a_{i}, a_{j}\right\}, a_{i} R(\vec{\succ}) a_{j}$ implies $a_{i} R\left(\vec{\succ}^{\prime}\right) a_{j}$.

Theorem 3 (Arrow's) If a SPF $R$ satisfies (AU) and (AIIA), then it is Arrow dictatorial.

Proof Given $R$, we can define a SCF $F^{R}$ that selects the alternative ranked highest by $R . F^{R}$ obviously satisfies (O), because by (AU), $F^{R}(\vec{\succ})=a_{i}$ if only $\left\{a_{i}\right\}$ is at the top. To see (CM), let $\vec{\succ}$ and $\{i, j\}$-runoff $\vec{\succ}^{\prime}$ agree on $\left\{a_{i}, a_{j}\right\}$. When $F^{R}(\vec{\succ})=a_{i}$, $a_{i} R(\vec{\succ}) a_{j}$, so by (AIIA), $a_{i} R\left(\vec{\succ}^{\prime}\right) a_{j}$. But $a_{i} R\left(\vec{\succ}^{\prime}\right) a_{k}$ for $k \notin\{i, j\}$ by (AU), so $F^{R}\left(\vec{\succ}^{\prime}\right)$ has to be $a_{i}$.

Theorem 1 thus presents a social choice dictator $n$ of $F^{R}$. She is a social preference dictator too. If $a_{i} \succ_{n} a_{j}$, individual $n$ dictates $F^{R}\left(T_{i j}(\vec{\succ})\right)=a_{i}$, so $a_{i} R\left(T_{i j}(\vec{\succ})\right) a_{j}$ and by (AIIA) $a_{i} R(\vec{\succ}) a_{j}$.

\section{Concluding remarks}

The simplicity is no coincidence. Our main theorem employs assumptions that are almost logically minimal (Yu 2013). Traditionally, people prove the GibbardSatterthwaite theorem as a corollary of the Muller-Satterthwaite theorem (Muller and Satterthwaite 1977). But the "monotonicity" condition assumed is more restrictive than (CM), making it a weaker theorem than ours. Reny (2001) has to circumvent the difficulty by giving parallel proofs of Arrow's theorem and the Muller-Satterthwaite 
theorem. Mas-Colell et al. (1995) prove Arrow's theorem first, but as a step toward the Muller-Satterthwaite theorem, the construction of $R^{F}$, a SPF derived from a SCF, is necessarily more involved than that of $F^{R}$. Building on these earlier works, ${ }^{2}$ our framework offers an alternative way of unifying and teaching classical theorems.

Acknowledgments I should thank Paul Milgrom and the students of Stanford Econ 204 (2013) for fieldtesting this novel way of teaching and learning social choice. This paper also benefited from comments by Kenneth Arrow, Matthew Jackson, David Kreps, Josh Mollner, Philip Reny, Larry Samuelson, Robert Wilson, Yiqing Xing, Constantine Yannelis, the editor Nicholas Yannelis, and seminar participants in Stanford University. I thank the support of Koret Foundation Stanford Graduate Fellowship and National Natural Science Foundation of China (71073102 and 71273171).

\section{References}

Arrow, K.J.: Social Choice and Individual Values, 2nd edn. Wiley, New York (1963)

Barberá, S.: Pivotal voters: a new proof of Arrow's theorem. Econ. Lett. 6(1), 13-16 (1980)

Geanakoplos, J.: Three brief proofs of Arrow's impossibility theorem. Econ. Theory 26(1), 211-215 (2005)

Gibbard, A.: Manipulation of voting schemes: a general result. Econometrica 41(4), 587-601 (1973)

Man, P., Takayama, S.: A unifying impossibility theorem. Econ. Theory (2013, in press). doi:10.1007/ s00199-013-0750-6

Mas-Colell, A., Whinston, M., Green, J.: Microeconomic Theory. Oxford University Press, Oxford (1995)

Muller, E., Satterthwaite, M.A.: The equivalence of strong positive association and strategy-proofness. J. Econ. Theory 14(2), 412-418 (1977)

Reny, P.J.: Arrow's theorem and the Gibbard-Satterthwaite theorem: a unified approach. Econ. Lett. 70(1), 99-105 (2001)

Satterthwaite, M.A.: Strategy-proofness and Arrow's conditions: existence and correspondence theorems for voting procedures and social welfare functions. J. Econ. Theory 10(2), 187-217 (1975)

Yu, N.N.: A one-shot proof of Arrow's impossibility theorem. Econ. Theory 50(2), 523-525 (2012)

Yu, N.N.: A quest for fundamental theorems of social choice. Working Paper (2013)

\footnotetext{
2 Another source of our inspiration is Man and Takayama (2013). They also derive other results from one main theorem, the proof of which is relatively long, partly because it works with neither SCFs nor SPFs.
} 